\title{
Auswahlregeln, erzeugende Operationen und zugehörige Punktmannigfaltigkeiten der Kristallstrukturen
}

\section{Doctoral Thesis}

Author(s):

Brandenberger, Ernst

Publication date:

1930

Permanent link:

https://doi.org/10.3929/ethz-a-000092434

Rights / license:

In Copyright - Non-Commercial Use Permitted 


\title{
Auswahlregeln, erzengende 0perationen und zugehörige Punktmannigfaltigkeiten der Kristallstrukturen
}

\author{
Von der Eidgenössischen Technischen Hochschule \\ in Zürich zur Erlangung der Würde eines \\ Doktors der Naturwissenschaften genehmigte \\ Promotionsarbeit
}

vorgelegt von

\section{Ernst Brandenberger}

aus Flazch (Kanton Zürich)

Nr. 621

Referent: Herr Professor Dr. P. Niggli

Korreferent: Herr Professor Dr. P. Scherrer

Akademische Verlagsgesellschaft m. b. H.

Leipzig 1930 


\section{Zusammenfassung.}

\$ 1. Diskussion der Auslöschungen einer allgemeinen Basisgruppe, welche auf die Unterscheidung von elementaren Auslöschungen, Kompositionsauslöschungen und Kompensationsauslöschungen führt. Die beiden ersten Gruppen von Auslöschungen sind einer allgemeinen, geometrischen Untersuchung zugänglich.

$\S$ 2. Charakterisierung der Auslőschungsgesetze. Die Beziehungen, welche unter den Basiskoordinaten notwendig sind, damit ein Auslöschungsgesetz zustande kommt. Bestimmt rationale Koordinaten, unbestimmt rationale und irrationale Koordinaten. Zähligkeit des Auslöschungsgesetzes, sein Geltungsbereich im Indizesfeld: nach $(h k l)$ integrale Auslöschungen, zonale und seriale Auslöschungen. Strukturtheoretische Deutung der Auslöschungen: die Frage nach den möglichen, erzeugenden Operationen.

§ 3. Die erzeugenden Operationen der elementaren Auslöschungen. Weg der systematischen Untersuchung der Symmetrieoperationen. Darstellung der Einzelfälle nach der Anzahl zur Operation variabler Koordinaten. Die geometrischen Örter der elementaren Auslöschungen und ihre Schnitte.

$\S$ 4. Die erzeugenden Operationen der Kompositionsauslöschungen. Geometrie der Kompositionen. Rationale Umgebung eines Punktes. Kennzeichnung der Kompositionen, die Erzeugende von Auslöschungen sind.

$\S$ 5. Diskussion nach $(h k l)$ integraler Auslöschungsgeșetze. Mögliche Operationen. Aussagen über die Basiskoordinaten. Mögliche Lokalisierung der Streuzentren aus Zähligkeit des Auslöschungsgesetzes und Anzahl der Streuzentren im Elementarparallelepiped.

§ 6. Diskussion zonaler Auslöschungsgesetze. Leitende Zonen und ihre Beziehungen zu den Symmetrieelementen. Auslöschungen in einer Zone als Auslöschungen eines ebenen Diskontinuums betrachtet. Aussagen über die Basiskoordinaten. Frage nach der erzeugenden Operation bei bekannter Klasse oder bei bekannter Lauesymmetrie.

§ 7. Diskussion serialer Auslöschungsgesetze. Leitende Ebenenserien und ihre Beziehungen zu den Symmetrieelementen. Leitende Ebenenserien in leitenden Zonen. Auslöschungen in einer Ebenenserie als Auslöschungen eines linearen Diskontinuums betrachtet. Aussagen über die Basiskoordinaten. Frage nach der erzeugenden Operation bei bekannter Klasse oder bei bekannter Lauesymmetrie.

§ 8. Die Bestimmung der Translationsgruppe. Wahl der Identitätsperioden. Äquivalenzen für die einzelnen, mehrfach primitiven Translationsgruppen. 
§ 9. Bestimmung des Raumsystems. Zonale Raumgruppenkriterien: Mögliche Form derselben und zugehörige, erzeugende Operationen. Diskussion der Äquivalenzen bei bekannter Kristallklasse oder bei bekannter Lauesymmetrie. Seriale Raumgruppenkriterien: Mögliche Form derselben und zugehörige, erzeugende Operationen. Diskussion der Äquivalenzen bei bekannter Klasse oder bei bekannter Lauesymmetrie. Zähligkeitsbeziehungen und Kompositionsmöglichkeiten. Ausschluß von Raumsystemen, wenn bestimmte Zähligkeiten nicht überschritten werden. Bestimmung der Basiskoordinaten.

§ 10. Pseudosymmetrieen. Innere Pseudosymmetrie. Äußere Pseudosymmetrie. In $^{\mathbf{n}}$ den relativen Lagebeziehungen und damit auch in den Auslöschungen übereinstimmende Strukturen. Zusätzlich in der Metrik und Topologie ähnliche Strukturen.

\section{$\S 1$. Das Selektionsverhalten einer allgemeinen Basisgruppe.}

Die Kriterien zur Bestimmung von Translationsgruppe und Raumsystem einer Kristallstruktur haben den Charakter notwendiger, aber nicht hinreichender Bedingungen Das von ihnen geforderte Verhalten einer Basisgruppe im Interferenzversuch kann, aber muß nicht den Nachweis für eine bestimmte Translationsgruppe oder ein bestimmtes Raumsystem leisten. Diese Eigenschaft der sogenannten $\gg$ kristallstrukturell wichtigen Auswahlregeln « verlangt, jedem Auslöschungsgesetz die Gesam theit der mit ihm verträglichen Basisgruppen und nicht nur einen Ausschnitt derselben zuzuordnen. Denn aus der Existenz eines bestimmten Auslöschungsgesetzes im Interferenzbild folgt als gesichert nur das, was allen diesen Basisgruppen gemeinsam ist. Schlußfolgerungen, die ohne Benutzung anderer Interferenzdaten weitergehen, verlassen den Boden strikter, strukturanalytischer Diskussion.

Die Diskussion der Auslöschungsgesetze im Hinblick auf ihre Mehrdeutigkeit ist daher für ihre strukturanalytische Verwertung von fundamentaler Bedeutung. Erst durch sie erhält die Methodik der Bestimmung von Translationsgruppe und Raumsystem jene systematische Grundlage, die den Abschluß ihres strukturtheoretischen Ausbaus darstellt. Die Frage nach der Mehrdeutigkeit der Auslöschungsgesetze vermag zudem prinzipielle Mängel der Strukturbestimmung miltels der Röntgeninterferenzen aufzudecken und so die Grenzen aufzuzeigen, die ihr in ihrer heutigen Form gesteckt sind. Die Untersuchung der Auslöschungsgesetze auf die Eindeutigkeit ihrer Aussagen hin wurde auf Anregung von P. Niggli aufgegriffen; in einer ersten Reihe von Arbeiten $\left.{ }^{1}\right)$ erfolgte die

1) E. Brandenberger und P. Niggli, Die systematische Darstellung der kristallstrukturell wichtigen Auswahlregeln. Z. Krist. 68, 301. 1928. - E. B randenberger, Systematische Darstellung der kristallstrukturell wichtigen Auswablregeln trikliner, monokliner und rhombischer Raumsysteme. Z. Krist. 68, 330. 1928. - E. B randenberger, Systematische Darstellung der kristallstrukturell wichtigen Auswahlregeln tetragonaler Raumsysteme. Z. Krist. 71, 452. 1929. - E.Brande n berger, Die strukturtheoretische Interpretation der Auswahlregeln. Schweiz. Min. u. Petr. Mitt. 8, 438. 1928. 